\title{
Christopher Daase
}

\section{Editorial}

Neben der großen Finanzkrise, die auf absehbare Zeit ein zentrales Thema der Internationalen Beziehungen sein wird, gibt es auch eine kleine Finanzkrise, die zumindest das Leben der ZIB nicht leichter gemacht hat.

\section{Neuer Vertrag mit dem Nomos-Verlag}

Mit dem satzungsgemäßen Auslaufen der zehnjährigen Förderung durch die Deutsche Forschungsgemeinschaft musste die Zusammenarbeit der ZIB mit dem NomosVerlag auf eine neue Grundlage gestellt werden. In zwei Verhandlungsrunden wurde ein neuer Vertrag ausgehandelt und mit den Herausgebern und dem Sektionsvorstand abgestimmt.

Die Neuerungen betreffen zum einen die so genannte Redaktionspauschale, mit der der Verlag die Redaktionsarbeit unterstützt. Sie beläuft sich auf 15\% des Nettojahresumsatzes, mindestens jedoch 3.000 Euro im Jahr. Damit wird zwar nur ein Bruchteil der Ausgaben abgedeckt, und die ZIB wird auf absehbare Zeit auf die Zuwendungen von einzelnen Instituten oder Lehrstühlen angewiesen bleiben. Gleichzeitig bietet die Formel aber einen Anreiz, die Verkaufszahlen der ZIB zu steigern. Jedes neue Abonnement kommt der Redaktionsarbeit direkt zugute.

Die zweite Neuerung betrifft den öffentlichen Zugriff auf die Texte der ZIB. Es ist vereinbart worden, dass alle Hefte nach zwei Jahren vollständig im Internet zugänglich gemacht werden. Bei Erscheinen eines neuen Heftes werden zwei Beiträge über die ZIB-Homepage kostenlos verfügbar gemacht. Nach einem Jahr können Autorinnen und Autoren vom Verlag eine PDF-Fassung ihrer Artikel erhalten, um sie auf ihre persönliche Homepage zu stellen.

\section{ZIB geht an die Bundeswehruniversität München}

Die ZIB geht in ihr letztes Jahr an der Ludwig-Maximilians-Universität München. Unter den genannten finanziellen Bedingungen war zu erwarten, dass es nicht leicht sein würde, einen Nachfolger für die Geschäftsführende Herausgeberschaft zu finden. Trotzdem gelang es, auf Vorschlag aus dem Sektionsvorstand und in Abstimmung mit den Herausgebern, Stephan Stetter und Carlo Masala für diese Aufgabe zu gewinnen. Da beide erst jüngst an die Universität der Bundeswehr in Neubiberg berufen wurden, zeigte sich diese großzügig und stellte, anders als seinerzeit die LMU, eine halbe Stelle für die Redaktion der Zeitschrift zur Verfügung. Im Sommer 
2009 wird der Transfer der ZIB an die UniBW in die Wege geleitet, sodass die Geschäftsführende Herausgeberschaft Anfang 2010 von einem neuen Team übernommen werden kann.

\section{Writing Foreign - ZIB-Roundtable auf der ISA}

Die ZIB-Redaktion hat mit einem Panel auf der diesjährigen ISA in San Francisco die Existenz und Problematik nicht-englischsprachiger IB-Zeitschriften ins Bewusstsein gerufen. Hintergrund unserer Initiative ist zum einen die immer wiederkehrende Diskussion in der deutschen Politikwissenschaft, insbesondere in der IB, ob es überhaupt noch zeitgemäß ist, wissenschaftlich auf Deutsch zu publizieren: Sollte man nicht gerade Nachwuchswissenschaftlerinnen und Nachwuchswissenschaftlern raten, mit ihren Aufsätzen lieber in einer der vielen englischsprachigen begutachteten Zeitschriften unterzukommen? Uns interessierte, ob auch in anderen nicht-englischsprachigen Ländern solche Diskussionen geführt werden und welche Argumente dort zu welchen Entscheidungen geführt haben. Zum anderen wollten wir generell auf die Problematik aufmerksam machen, die durch die Bevorzugung englischsprachiger Zeitschriften im Social Science Citation Index (SSCI) entstehen. Nicht-englischsprachige Zeitschriften haben es nämlich deutlich schwerer als englischsprachige, in den SSCI aufgenommen zu werden. Da aber die Kriterien für die Aufnahme durch die Firma Thomson Reuters vollkommen intransparent sind und die Ablehnung, wie im Falle der ZIB geschehen, keiner weiteren Begründung bedarf, ist eine unabhängige Überprüfung der Entscheidungen oder gar eine Revision nicht möglich. Dass private Standardsetzung zu Wettbewerbsverzerrungen führen kann, ist ein bekanntes Phänomen der Governanceforschung. Tatsache ist jedenfalls, dass es im Zuge der wissenschaftlichen Globalisierung tendenziell weniger attraktiv wird, in nicht-englischsprachigen Zeitschriften zu publizieren, weil inzwischen auch in nicht-englischsprachigen Ländern der SSCI-Index als Goldstandard wissenschaftlicher Exzellenz gilt und Berufungs- sowie Gehaltsverhandlungen auf Grundlage des SSCI geführt werden. Die Frage ist also, welche Berechtigung (und Wertigkeit!) das Publizieren in anderen Sprachen als Englisch heute hat und zukünftig haben soll. Und wie kann ein möglichst globaler Wissenschaftsdiskurs geführt werden, ohne dass nationale oder regionale Wissenschaftskulturen marginalisiert werden?

Diese Fragen fanden unter den Eingeladenen (u. a. Didier Bigo von Culture et Conflit, Gordon Mace von Etudes Internationales und Sonia de Camargo von Contexto Internacional) großes Interesse. Einigkeit bestand darin, dass die ISA neben ihrer Funktion als Plattform für den globalen Wissensaustausch sich stärker als bisher für die Förderung regionaler Wissenschaftskulturen im Bereich der Internationalen Beziehungen einsetzen müsse. Es wurde eine stärkere Kooperation zwischen den nicht-englischsprachigen Zeitschriften verabredet mit dem Ziel, den Sinn für die Vielfalt der Ansätze und Ansichten wach zu halten und eine gemeinsame Stimme gegenüber der ISA und Thomson Reuters zu bilden. Für die ISA 2009 ist ein zweiter 
Roundtable angenommen worden, an dem die Herausgeber weiterer nicht-englischsprachiger Zeitschriften (u. a. Politika, Internasjonal Politikk, Kokusai Seiji, CONfines) teilnehmen.

\section{ZIB-Panel auf der Deutsch-Britischen IB-Tagung in Arnoldshain}

Auf der diesjährigen deutsch-britischen IB-Tagung in Arnoldshain wurde ein ZIBPanel veranstaltet. Die Idee war, die unterschiedlichen Wissenschaftskulturen zu kontrastieren und zu fragen, warum es in Großbritannien eine eigene „Englische Schule" gibt, wohingegen sich die Mehrzahl der Forscherinnen und Forscher in Deutschland eher dem amerikanischen Mainstream verpflichtet fühlt. Neben der Pfadabhängigkeit der unterschiedlichen Entwicklungen wurde auf den unterschiedlichen wirtschaftlichen Stellenwert der IB in beiden Ländern hingewiesen. Während die IB in Deutschland nach wie vor ein Teilgebiet der Politikwissenschaft ist, ist sie in Großbritannien aufgrund des „Markterfolgs“ (Chris Brown) vor allem unter overseas students zu einer eigenständigen Größe innerhalb der sozialwissenschaftlichen Institute und Fakultäten geworden. Auch wenn sich die wenigsten britischen Wissenschaftlerinnen und Wissenschaftler der Englischen Schule zugehörig fühlen, gibt es nach Auffassung der Panelteilnehmer ein Bewusstsein, anders als der amerikanische Mainstream zu arbeiten. Einen ausführlichen Bericht dieser Tagung können Sie auf Seite 303 dieses Heftes finden.

\section{Online-Arbeitspapiere im Begutachtungsverfahren}

Im Laufe dieses Jahres ist die Frage aufgetreten, ob eine Veränderung des bewährten ZIB-Begutachtungsverfahrens notwendig ist. Da insbesondere von Forschenden, die über Drittmittel finanziert werden, zunehmend verlangt wird, im Sinne von „open access" ihre Forschungsergebnisse möglichst schnell online als Arbeitspapiere zu publizieren, ergeben sich Schwierigkeiten bei der Begutachtung solcher Beiträge in einem double-blind peer-review-Verfahren, wie es die ZIB vorsieht. Die Redaktion ist zu dem Schluss gelangt - gemäß der international üblichen Praxis -, nicht länger zu verlangen, dass Texte vom Netz genommen werden müssen, bevor sie in das Begutachtungsverfahren eingespeist werden. Artikel im Begutachtungsverfahren können online bleiben. Wenn das Manuskript erfolgreich ist, sollte der Onlinetext durch einen Link zum publizierten ZIB-Artikel ersetzt werden. Bei diesem Vorgehen muss allerdings in Kauf genommen werden, dass die Anonymität des begutachteten Manuskripts von der Redaktion nicht mehr garantiert werden kann. 


\section{Dank an Redakteure}

Abschließend sei an dieser Stelle zwei vor kurzem ausgeschiedenen Redakteuren der ZIB gedankt: Sebastian Schindler und Alexander Heppt. Sebastian Schindler übernahm den Posten des Chefredakteurs gleich nachdem die ZIB nach München gekommen war und führte überaus effiziente Arbeitsabläufe ein. Alexander Heppt übernahm seine Aufgabe für die letzten beiden Jahre und leitete das ZIB-Büro mit großem Enthusiasmus. Nicht zuletzt diesen beiden studentischen Redakteuren ist es zu verdanken, dass die ZIB auch ohne hauptamtliche Redakteursstelle ihre wissenschaftliche Qualität und redaktionelle Professionalität erhalten konnte. 